de Masaccio de Sta. María Novella. El frontón está sostenido por una pareja de columnas de cristal, como las de la parte inferior. A los lados vuelven a aparecer dos cuerpos oblicuos con sus diminutas hornacinas rematadas en carátulas y en su interior otras dos efigies de santos que a simple vista parecen piezas más interesantes que las de la zona inferior. Muy posiblemente realizados en plata, la figura de la izquierda parece representar a San Pablo, mientras que la derecha, intensamente movida con un movimiento un tanto acaracolado muy manierista, podría representar a San Andrés. Los otros dos cuerpos laterales muestran sendos pájaros posados en una rama de naranjo con flor de azahar y un rojo fruto, en un motivo de gran tradición en este tipo de trabajo que ha pervivido hastas nuestros mismos días. Curiosamente estos dos cuerpos aparecen enmarcados por sendas columnas salomónicas con los típicos capiteles en bronce ya vistos pero en este caso trabajadas no en cristal sino en plata. Finalmente, el mueble remata en un frontón partido en los cuerpos exteriores y con balaustrada los cuerpecillos intermedios, realizadas en plata los diminutos balaustres y no en cristal como en la zona inferior.

Conviene también destacar la decoración de los laterales del mueble en los que vemos sendos tableros de ébano y piedras duras, con una ágata en la parte central de considerables dimensiones flanqueada por dos pilastras con capitel de bronce. En estos espacios laterales se van siguiendo la base y zonas del resto del mueble, rematando en cornisa de balaustrillos de plata.

La pieza podemos situarla con bastante precisión en los años finales del siglo XVI o muy en los comienzos del XVII y todo apunta a ser obra de las manufacturas florentinas de esas fechas. Ver las relaciones y parecidos con piezas conservadas sería trabajo largo e incluso carente de sentido. Ejemplares que muestran un gran parecido con esta pieza de Torrijos los tenemos muy claros en el Museo degli Argenti de Florencia, en el Kunsthistorisches Museum de Viena y en infinidad de otros museos y colecciones privadas. Y como una simple prueba de que determinados motivos perviven largamente, como ya hemos destacado, queremos dejar constancia del hecho de que piezas florales que aparecen en este mueble siguen trabajándose exactamente iguales en el lateral del mueble de Joseph Baumhaver, de entrado el siglo XVIII, conservado en el Palacio de Versalles ${ }^{7}$.

De cómo pudo llegar pieza tan espléndida a esta villa de Torrijos, el camino creemos verlo claro, ya que esta población fue posesión de la familia Cárdenas, luego Duques de Maqueda, algunos de cuyos miembros sirvieron al rey de España en Italia e incluso la Dra. Rosa López Torrijos ha podido documentar el encargo y envío de piezas italianas hacia esta villa en $1668^{8}$.

Juan Nicolau Castro

Dr. por la Universidad Autónoma de Madrid

\title{
LO QUE VIO EN 1636 (DE ARQUITECTURA Y ARTES DECORATIVAS) EN ESPAÑA UN VIAJERO CHECO
}

No hay muchos testimonios directos que documenten de primera mano las opiniones de los viajeros checos a España en el siglo XVII, y mucho menos que tuvieran provecho para la historia del arte, aunque perfectamente podría escribirse un capítulo más en los libros exis-

\footnotetext{
7 Anna Maria Giusti: Idem.

${ }^{8}$ Mercedes Gómez-Ferrer Lozano: «El taller escultórico de Juan de Lugano y Francisco de Aprile en Valencia», Universitat de València, 1997.
} 
tentes ${ }^{1}$. Sí tenemos, por ejemplo, un documento excepcional que describe, en el siglo xv, España y su gente, su paisaje, ciudades y templos, así como obras de arte ya desaparecidas ${ }^{2}$. Pero hasta ahora se ha prestado poca atención a las siguientes centurias, la del XVI, cuando algunos nobles del Reino de Bohemia (por ejemplo, Pernestán y Dietristein) casan con damas españolas de alto rango ${ }^{3}$, y, sobre todo, al siglo xviI, aunque para este último existan documentos incluso publicados. Lo que los historiadores «puros» consideran una desventaja —el hecho de que prevalezcan descripciones más de obras de arte que comentarios a la situación política o económica-, los historiadores de arte acogen con el mayor interés, aunque se trate de descripciones secas, más turísticas que analíticas. Éste es el caso de dos testimonios publicados hace tiempo del caballero Kounic ${ }^{4}$.

Su importancia consiste no tanto en los mismos apuntes de viaje que aquí presento a manera de muestra, sino en el hecho de que Lev Vilém z Kounic (Leo Guillermo de Kounicz) fundó, quizás inspirado por lo que había visto en Italia y en España, la pinacoteca familiar de Slavkov (sitio conocido más por Austerlitz, por labatalla de Napoleón). Lamentablemente, las ventas a principio del siglo xIX y un incendio en su final, en 1897, empobrecieron a la pinacoteca, en la cual se guardaban, entre otras, obras españolas, por ejemplo de Alonso Cano ${ }^{5}$. Según la lista del año 1818, uno de los cuadros fue atribuido a Claudio Coello (hoy figura como obra de Mateo Cerezo, en la colección vienesa de los Černín), el segundo, obra de Antonio Pereda, fue vendido hace decenios en la tienda del anticuario V. Hořejš de Praga. También figuraba en la colección de Slavkov (p. 40) una Huida a Egipto, cuyo autor fue el pintor Ximenes, activo en Brno hacia $1700^{6}$.

Con el tiempo, cuando varios de los Kounic ocuparon puestos de embajadores en Madrid, entraron en la colección Goya, El Greco, Murillo, Ribera y Velázquez ${ }^{7}$. Según advierte la investigadora húngara Harászti-Takács ${ }^{8}$ comenzando por el famoso canciller checo de María Teresa Wenceslao Kaunitz (Václav Antonín z Kounic) varios miembros de la estirpe se dedicaron al servicio diplomático precisamente en Madrid, lugar con el que mantuvieron buenas relaciones incluso «fuera de servicio»: Domingo Antonio (Dominik Antonín) como primero entre 1774 y 1779, luego Wenceslao José Clemente (Václav Josef Klemens), enterrado en Barcelona en 1785. Le siguió Luis Wenceslao (Alois Václav) hasta 1806, quien pasó una temporada en Madrid aún en el período de 1815 a 1816. Lo que es digno de atención es que compraba obras de un artista contemporáneo, desconocido, a la sazón, en Viena: Goya, lo cual puede apreciarse por la des-

\footnotetext{
1 María de los Santos García Felguera, Viajeros, eruditos y artistas. Los europeos ante la pintura española del Siglo de Oro. Madrid, 1991.

2 Véase Jiři Hrubeš, «El itinerario checo más antiguo de España y Portugal». Ibero-Americana Pragensia (Praga), V, 1971, pp. 69-82.

3 Véanse P. Štěpánek - E. Bukolská, «Retratos españoles en la colección Lobkowitz en Roudnice». Archivo Español de Arte XLVI, 1973, s. 319-339; Ibid., «Retratos españoles en la colección Lobkowicz, en Roudnice». I. Ibero-Americana Pragensia VI, 1972, s. 145-162, etc.

${ }^{4}$ František Hrubý. Lev Vilém z Kounic. Barokní kavalír. (Leo Guillermo de Kounic, caballero barroco). Edición preparada por Libuše Urbánkova-Hrubá. Státní oblastní archív v Brně (Archivo de Estado Regional de Brno) 1987. Un extracto aparece en checo también en el libro de Ema Urbánková, Česká touha cestovatelská (El anhelo de viajar checo). Praha 1988, s. 165-166. Luego, en castellano, en parte, por Simona Binková y Josef Polišenský, «Dos testimonios sobre España del siglo xvII», Ibero-Americana Pragensia (Praga), XXIII, 1989, pp. 131-152, de donde saco las citaciones referentes al arte y arquitectura exclusivamente. Sin embargo, la primera que estudió el viaje español de Kounic fue Cecile Hálová-Jahodová, «Galerie moravských Kouniců», Časopis Matice moravské, roč. 63-64, Brno, 1940, separata, pp. 3 y ss.

5 C. Hálova-Jahodová, op. cit., en la nota 4, p. 4, se le da la forma de «Caimo», o por error de lectura o de tipografía.

${ }^{6}$ Véase P. Štěpánek, «Celestino (?) Ximenes, un pintor español ignorado». Archivo Español de Arte, Madrid, 1972, n. ${ }^{\circ}$ 177, pp. 61-63, il. Dado que hay nuevos materiales sobre él que presentaré en un artículo aparte, aprovecho la ocasión para dar a saber que habrá que borrar el signo de interrogación de su nombre Celestino Ximenes.

7 C. Hálová-Jahodová, op. cit. en la nota 4, pp. 75-80.

${ }^{8}$ Marianne Harászti-Takács, «Scénes de genre de Goya á la vente de la collection Kaunitz en 1820». Bulletin du Musée Hongrois des Beaux-Arts, n. ${ }^{\circ} 44$, pp. 107-121.
} 
cripción y las cuentas del año 1822. De la subasta de Viena, los cuadros pasaron a Budapest y a otras colecciones en el mundo y algunos terminaron allende del océano.

Hay que decir que los caballeros checos tuvieron la obligación de conocer en primer lugar Italia y luego Europa occidental, y a partir del s. xvi, sobre todo en el momento de la contrarreforma en Bohemia que le hizo volver al catolicismo, también España. Ya hice mención del famoso caballero Sporck, del s. XVIII ${ }^{9}$, quien en vez de dejar un testimonio por escrito, lo dejó en sus obras, inspirándose tanto por Montserrat como por El Escorial en su residencia que construiría más tarde en Kuks, joya del barroco universal.

Ahora bien, en el siglo xvir se intensifican los viajes de caballeros, casi La Grande Tour para completar su educación y experiencia de los jóvenes caballeros que conocen Europa antes de ocupar puestos de rango, y, algunos de ellos, dejan un interesante testimonio, escrito en forma de apuntes diarios. Uno de los casos ejemplares es el de Leo Guillermo de Kpunic Kaunitz (Lev Vilém z Kounic, 1614-1655), quien emprende su viaje en 1636, y el otro, Germán Jacobo Černín (Heřman Jakub Černín, 1659-1710) en los años ochenta, caso que voy a tratar en otra oportunidad.

A manera de presentar al protagonista, sólo puedo aportar unos datos para no abultar y exagerar la parte histórica: Leo Guillermo de Kounice perteneció a una de las estirpes más importantes de Moravia. Siendo de corta edad, no compartió el destino de otros parientes suyos calvinistas — salir del exilio después de la perdida batalla en la Montaña Blanca (1620)—, sino que fue conferido a la educación del cardenal Francisco de Dietrichstein, obispo y gobernador de Moavia residente en Olomouc, quien logró convertir al niño al catolicismo y luego lo mandó a estudiar a colegios jesuitas. Al cumplir el joven con sus obligaciones y llegar a la mayoría de edad, el cardenal lo envió a un viaje a los países católicos: Itali (incluida Sicilia), Malta y España. Dados los contactos que tenía éste con el medio español (su madre fue Margarita de Cardona y Recansens, enterrada en la catedral de San Vito de Praga). Más tarde, en 1637, emprendió otro viaje a los países protestantes: Inglaterra, los Países Bajos y Alemania. En su segundo matrimonio casó con la sobrina del cardenal, Eleonora de Dietrichstein, ocupando con el tiempo el cargo de juez mayor de Moravia.

Aunque el diario del viaje a España en 1636 resulta ser un fragmento, queda todavía una buena fuente de informaciones apuntadas en alemán, menos en checo, en español o cifrado. Procedente de Génova, llega a Alicante el 27 de agosto de 1636, siguiendo por Albacete y Aranjuez a Madrid, a donde llega el 7 de septiembre. Después de buscar alojamiento, mandó coser para sí mismo y para las personas de su servicio trajes a la moda española. Iba a oír misas a diferentes iglesias de la capital y a pasear al Prado, tomando clases del español y del esgrima. En otras oportunidades se entretenía con la alquimia, iba a ver comedias a los teatros Príncipe de Corral y Corral de la Cruz, paseaba y asistía a las ceremonias de la Corte.

El 21 de septiembre por la mañana visitó la Capilla Real: «Allí todos los caballeros lucían cadenas de oro como en días de fiestas importantes». Por la tarde vio al rey pasar «públicamente y con gran solemnidad, junto con la reina (o sea, Felipe IV e Isabel) y el joven Infante, dirigiéndose a Nuestra Señora de Atocha. Primero pasó un desfile de carrozas que eran alrededor de ocho mil. Luego siguió un coche tirado por seis caballos, bellísimo, igual que la silla de manos del Rey. Luego cabalgaron varios centenares de caballeros en los caballos más hermosos que puedan verse y luego príncipes y grandes y luego la carroza de la Reina en la que la Reina y el Infante iban solos. Al lado del coche, a la derecha, cabalgó el Rey en medias y zapatos, conversando con la Reina...».

Al visitar, el día 4 de octubre, el noviciado de los jesuitas, luego a los franciscanos descal-

\footnotetext{
${ }_{9}$ «Una corte provinciana de alcance europeo - Kuks». In: El arte en las cortes europeas del siglo xvIII. Madrid, 1987.
} 
zos (San Francisco); para dejarle la palabra al joven checo, «luego me fui a San Felipe, donde la orden de los Caballeros de Santiago ofrecía una misa solemne. Allí, alrededor del claustro, colgaron preciosos tapices bordados de seda y oro»...

El 5 de octubre... «me fui a Santo tomás, un convento de dominicos no muy grande. Allí vi la larga, estrecha y puntiaguda estandarte que tuvo don Juan de Austria en la lucha contra los turcos. Estaba hecho de damasco azul con cuatro escudos, en medio del escudo el Papa Pablo $\mathrm{V}$, a la derecha el Emperador, a la izquierda el de los venecianos y bajo el del Papa estuvieron las armas de Castilla. Nota: $\mathrm{Y}$ sobre todos los escudos estaba pintado un gran crucifijo. En la iglesia hubo una pequeña estandarte más, cuadrangular, de color del clavo de olor, también decorada. De allí me fui a la iglesia de la Santa Trinidad, vecina la Santo Tomás ... Por la tarde ... a la Casa de Campo, un palacete del Rey con un jardín. Justamente a la entrada se encuentra la estatua del Rey Felipe III, padre del Rey actual, una gran estatua ecuestre de metal que le regaló y mandó el duque de Florencia. De allí volvimos a través de un puente bello a Prado donde hubo una gran cantidad de coches».

Los días 15 al 17 de octubre los pasó en Toledo. «Aquí primero vimos el bonito hospital de San Juan Bautista que mandó construir el cardenal don Juan de Tavera, arzobispo de Toledo. El 16 por la mañana fui al domo arzobispal, muy bello y tan bien construido como el domo de Milán. Allí en la Capilla mozárabe, construida y decorada por orden del cardenal Francisco Jiménez de Cisneros, por primera vez oí dos misas llamadas mozárabes... después... fui a ver dentro de la iglesia un altar donde en una piedra se conservan las huellas de la Virgen cuando le imponía a San Ildefonso la Casulla. Luego vimos el coro de canónigos colocado en medio de la iglesia. En la parte opuesta, en el extremo de la iglesia, se encuentra el retablo mayor, arquitectónicamente concebido como el coro en la capilla. Luego, en la sacristía vimos un sagrario y en él un tesoro extraordinario igual al que no he visto nunca en una iglesia, fue mayor al de Loreto. Entre otras cosas hubo allí ante todo un vestido para la estatua de la Virgen en la Capilla, todo cubierto de perlas, diamantes y otras semejantes piedras preciosas de mucho valor, con tanta densidad que no se pudo ver el fondo en absoluto. Este se evalúa a 100.000 coronas.

También vimos el utensilio de plata que aquí suelen usar al celebrar la fiesta de Santo Sepulcro. Vimos también el cuerpo de Santa Leocadia en un hermoso ataúd de plata y en una arqueta de oro la moneda por la cual Nuestro Señor y Salvador fue vendido. Nota bene: Y por fin, un sinnúmero de reliquias, cruces de oro, etc. La plata, por ser tan abundante, no está apreciada mucho aquí. Nota bene: Luego vimos un pequeño retablo con figuras como si exaltadas, hechas de puras piedras preciosas. De aquí pasamos a una sacristía más pequeña donde los curas se preparan para la misa. Aquí, en un armario, estuvo colocado un relicario con un pedacito de madera en medio, procedente de la Santa Cruz, regalo de San Luis, Rey de Francia. En otro armario se conservaban cuatro volúmenes muy bien escritos e iluminados de la Escritura Santa por los que el Rey estuvo dispuesto a dar una ciudad entera. En otros armarios vimos una gran cantidad de ínfulas bordadas con perlas, una corona de oro de la Virgen cubierta por piedras preciosas de gran valor y arriba con una bola hecha de un solo pedazo de una bella esmeralda oriental. Tan sólo el trabajo de orfebre en esta corona había costado 14.000 coronas. Luego hubo aquí dos pulseras de oro de la Virgen cubiertas por piedras preciosas y también esta vez sólo el trabajo costaba 4.000 coronas. Item varios cálices de oro cubiertos de perlas cuya sombra formaba dos dibujos. Item un relicario de lazulita, cubierto de piedras preciosas en medio con un pedacito de madera de la Santa Cruz sobre la que juran los Reyes a los privilegios de los toledanos y muchas cruces semejantes con una cantidad enorme de piedras preciosas. Por fin vimos una custodia de plata de altura de dos hombres, dorada, muy bien trabajada. Había en ella - tan sólo de plata- 450 libras. En medio de ésta hubo otra custodia, más 
pequeña, de puro oro muy bien labrado y es precisamente el trabajo lo que más vale en ella. El oro utilizado para la custodia pesa 50 libras. Nota: Alrededor de la custodia más pequeña hay pequeñas campanillas.

En la cercanía de esta sacristía hay un espacio lleno de sacristías pequeñas con una cantidad de paramentos de iglesia y antipendios. Nota: De allí pasamos a una capilla muy hermosa que mandó construir de mármol el cardenal don Bernardo de Rotas (¿Rojas?). En la capilla hay una imagen milagrosa de Nuestra Señora tallada en madera colocada libremente bajo el arbotante. De allí nos fuimos a otra capilla bajo el altar mayor donde se guarda el cuerpo de San Eugenio, el primer arzobispo de Toledo, y luego pasamos a la sala capitular; allí se hallaba un bello artesonado de rica talla bien dorado y alrededor se encontraban retratos de todos los arzobispos. Y todo eso lo mandó hacer el cardenal Rotas para asegurarse un lugar en la Capilla Mozárabe.

De allí subí a la torre de la iglesia y admiraba la grandeza y la posición de la ciudad. Después visité el hospital de los locos... Por la tarde... fui con algunos españoles al Palacio Real, el Alcázar. Lo vi todo. Tres cosas en él merecen atención: en primer lugar, es una bella y ancha escalera de rara invención, la más bonita que haya visto en mi vida, parecida a un teatro, en el primer plano con una hermosa capilla. La segunda cosa en una doble, bella y larga galería en la parte más alta del palacio. La tercera son las caballerizas subterráneas, bonitas y grandes, que se encuentran alrededor del palacio. También la cochera es interesante por pasar para abajo. Nota: De afuera se entra al palacio directamente por una puerta; sin embargo, del palacio para afuera lleva una escalera la que es posible subir y bajar montando un caballo.

El 17 de octubre... luego fue al acueducto que conduce el agua muy diestramente desde abajo, del río, hasta para arriba al palacio de dos maneras diferentes -el primero modo más antiguo, es el de Juanelo - ... Por la tarde visité el convento de San Juan de los Reyes. ... Es un hermoso convento que mandó a construir la Reina y de mayor belleza allí son el claustro y la iglesia. En las fachadas de esta última fue colocado un gran número de cadenas en las que ... fueron encadenados los cristianos por los moros en el tiempo cuando aquellos conquistaron Toledo. De allí me fui a la catedral para volver a ver el sagrario.

A la vuelta compré unas 1.000 agujas y después me iba preparando para el viaje de regreso ... a Madrid con una posta...»

Lamentablemente, no se dan detalles de la visita de Kounic al palacio Real de Madrid donde vio su guardajoyas y su armería, y tampoco del palacete de El Pardo; sólo se comenta la visita al Escorial el día 27 de octubre, con una extraordinaria riqueza de detalles. Dado que a veces se trata de una descripción pasiva, no transcribo todo, sino sólo extractos: «San Lorenzo que suele considerarse, con toda la razón, como la octava maravilla del mundo. Allí fui primero a oír la misa. Luego fui al Palacio Real y allí vi al Rey jugar la pelota. Después el marqués de las Fuentes y el mayordomo me invitaron a comer a la mesa de los gentilhombres de la corte. Por la tarde, un caballero de la Orden de Santiago me acompañó para mostrarme todos los edificios. Primero fuimos a ver el convento de los jerónimos... En el centro del primer claustro grande se halla una hermosa fuente construida a modo de un arco triunfal en cuyas cuatro esquinas hay otras fuentes adornadas por los escudos de cada de los cuatro evangelistas. ... en el coro de los monjes hubo una bonita instalación de latón dorado en que se apoyaban los libros del coro con los que la gran instalación movía y los volteaba con facilidad. ... Luego vimos la hermosa sacristía donde se guardaban paramentos de todo tipo, todos del mismo color y de la misma forma para todos los altares de los que hubo 40 en la iglesia, para todas las épocas del año. Entre otros, vimos varios paramentos con diseño figural, bordados de oro y plata de todos los colores, labrados «con el labor matizato». Además, vimos otros paramentos tejidos de oro y cubiertos de perlas, de un gran valor. Por fin, hubo allí 40 altos cande- 
labros de plata destinados para ser usados en las procesiones. Nota: Los armarios de la sacristía, igual que los loibros del coro ya mencionados, tenían abajo ruedas para que, siendo tan grandes, sea más fácil moverlos. ... El exterior de la iglesia parecía al de la catedral de San Pedro en Roma siendo algo más pequeña... El más extraordinario de la iglesia es el retablo mayor: está hecho de jaspe, con doce columnas de la misma piedra, tiene doce grandes estatuas de latón dorados de los doce Santos Apóstoles de la Virgen. En medio del altar hay un tabernáculo o ciborio donde se guarda el Santísimo Sacramento. Este ciborio es por afuera de jaspe y de latón dorado, con diez columnas de gran valor, también de jaspe - el trabajo costó 10.000 ducados. En el interior del tabernáculo hay otro ciborio pequeño, todo de oro y piedras preciosas. Hasta en éste está guardado el Santísimo Sacramento. Directamente al lado del alto altar se encuentra el ostensorio del Rey y encima de él las estatuas de latón dorado del Emperador Carlos V y su esposa a la derecha y en frente las estatuas de Felipe II y su esposa a la izquierda. Y al lado de estas estatuas se hallan semejantes columnas de jaspe como en el altar alto. En los extremos más distantes de la iglesia se encuentran en ambos lados unos órganos de sumo valor... Nota: para mantener la limpieza tanto en el coro, como en la iglesia, suelen poner al suelo redondas esteras en las que esculpen. En la capilla de la iglesia hay una cruz muy diestramente confeccionada de cartón...».

Visitó las bibliotecas donde observó, entre otras cosas, los retratos de Carlos V y de los Felipes, y el Palacio Real, «donde nos mostraron todas las galerías, salas y habitaciones del Rey, hasta su lecho en el que suele dormir. Después regresamos a casa en un carruaje tirado por seis mulas del Rey.»

Al día siguiente, Kounic visitó la academia y el colegio de seminaristas de El Escorial, la iglesia, donde observó un gran número de reliquias de diferentes hombres y mujeres santos, a una pieza por encima de la sacristía «lleno de cosas preciosas y antigüedades; como p. ej. una de las siete vasijas en la que Nuestro Señor y Salvador transformó el agua en vino, luego una cruz bordada de perlas por la misma Emperatriz Helena, una parte de la parrilla de San Lorenzo junto con un pedacito de su piel, o un pequeño altar que trajo consigo Carlos V».

Aunque el joven Kounitz se limita a descripciones bastante pasivas, sus notas en el diario de viaje nos resultan útiles para saber qué es lo que fue accesible a un joven que luego podía materializar de alguna forma sus recuerdos de viaje.

PAVEL ŠTĚPÁNEK

Dr. en Historia del Arte, Universita Palackého, Olomouc 